\title{
Enhancement of cytotoxic and pro-apoptotic effects of 2-aminophenoxazine-3-one on the rat hepatocellular carcinoma cell line dRLh-84, the human hepatocellular carcinoma cell line HepG2, and the rat normal hepatocellular cell line RLN-10 in combination with 2-deoxy-D-glucose
}

\author{
AKIRA TAKEMURA $^{1 *}$, XIAO-FANG CHE $^{2 *}$, TAKAFUMI TABUCHI $^{1}$, \\ SHOTA MORIYA $^{2}$, KEISUKE MIYAZAWA ${ }^{2}$ and AKIO TOMODA ${ }^{2}$ \\ ${ }^{1}$ Fourth Department of Surgery, Tokyo Medical University, Ibaraki Medical Center, 3-20-1 Chuo, Inashiki, Ibaraki \\ 300-0395; ${ }^{2}$ Department of Biochemistry, Tokyo Medical University, 6-1-1 Shinjuku, Tokyo 160-8402, Japan
}

Received August 15, 2011; Accepted October 3, 2011

DOI: $10.3892 /$ or.2011.1531

\begin{abstract}
The cytotoxic and pro-apoptotic effects of a single dose of 2-aminophenoxazine-3-one (Phx-3) or 2-deoxyglucose (2-DG) or of a combined dose of Phx-3 and 2-DG were studied in the rat hepatocellular carcinoma cell line dRLh-84, the human hepatocellular carcinoma cell line HepG2 and the rat normal hepatocellular cell line RLN-10. The number of viable cells decreased in a dose-dependent manner, when dRLh-84, HepG2 or RLN-10 cells were treated with 2-DG $(0.5-20 \mathrm{mM})$ or Phx-3 (1-50 $\mu \mathrm{M})$ alone at $37^{\circ} \mathrm{C}$ for $48 \mathrm{~h}$. When these cells were treated with $10 \mathrm{mM}$ 2-DG and different concentrations of $\mathrm{Phx}-3$, the number of viable cells decreased dose-dependently and in an additive manner for these agents. A single dose of 2 or $10 \mu \mathrm{M}$ Phx-3 induced apoptotic morphology characterized by nuclear condensation and cell shrinkage in dRLh-84, HepG2 and RLN-10 cells, while a single dose of $10 \mathrm{mM}$ 2-DG did not. When Phx-3 (2 or $10 \mu \mathrm{M})$ treatment was combined with 2-DG $(10 \mathrm{mM})$ treatment in these three cell lines, the cells with apoptotic morphology increased extensively, which was confirmed by flow cytometric analysis. In addition, autophagic morphology characterized by cytosolic vacuole formation was significantly increased in the hepatocellular carcinoma cell lines dRLh-84 and HepG2 but not in the normal hepatocel-
\end{abstract}

Correspondence to: Dr Akio Tomoda, Department of Biochemistry, Tokyo Medical University, 6-1-1 Shinjuku, Tokyo 160-8402, Japan E-mail: tomoda@tokyo-med.ac.jp

*Contributed equally

Key words: 2-aminophenoxazine-3-one, 2-deoxy-D-glucose, hepatocellular carcinoma cells, normal hepatocellular cells, pro-apoptotic effect lular cell line RLN-10 after a single dose of Phx-3 or 2-DG or a combined dose of Phx-3 and 2-DG. Furthermore, when dRLh-84 and HepG2 cells were treated with Phx-3 alone or a combined dose of Phx-3 and 2-DG, depolarization of the mitochondria was extensive, but that of the normal cell line RLN-10 was not. These results may imply that the mechanism for the apoptosis of hepatocellular carcinoma cells caused by $\mathrm{Phx}-3$ alone or a combined dose of Phx-3 and 2-DG differs from that of the normal cell line RLN-10. The present results demonstrate that Phx-3 alone may be beneficial for targeting liver cancer and that its anticancer activity may be enhanced by 2-DG. However, a combined dose of Phx-3 and 2-DG may exert adverse effects on normal liver cells, as evidenced by the cytotoxic and pro-apoptotic effects of the combined treatment in the rat normal hepatocellular cell line RLN-10.

\section{Introduction}

Cancer cells are generally characterized by paradoxical behavior recognized as the Warburg effect (1), i.e., augmented rates of glycolysis and suppressed mitochondrial oxidative phosphorylation under aerobic conditions in these cells. Therefore, suppression of glycolytic metabolism in malignant cells has been a target of cancer treatment (2-4). Since a low intracellular $\mathrm{pH}(\mathrm{pHi})$ inhibits the activity of glycolytic enzymes, including phosphofructokinase in both normal cells and in cancer cells $(5,6)$, leading to suppression of the glycolytic metabolism, it is reasonable that agents that reduce $\mathrm{pHi}$ may be candidates for cancer treatment (7-9). Amiloride and its derivative, 5-(N,N-hexamethylene) amiloride (HMA) have been shown to decrease $\mathrm{pHi}$ in cancer cells, thereby exerting anticancer activity $(10,11)$. Che and colleagues (12-15) have demonstrated that an oxidative phenoxazine, 2-aminophenoxazine-3-one (Phx-3) exerts cytotoxic effects against a variety of cancer cells and causes apoptosis of these cells in vitro, by reducing $\mathrm{pHi}$ in these cells and thereby perturbing the intracellular homeostasis, including the glycolytic metabolism. 
Another measure to prevent glycolysis in normal cells and cancer cells is to use 2-deoxy-D-glucose (2-DG), which has been shown to suppress the glycolytic metabolism in these cells, due to the specific inhibition of hexokinase, an initial rate-limiting enzyme of the glycolytic pathway (16), and therefore, has been applied to animal model studies and human clinical trials for cancer treatment (17,18). 2-DG itself does not exert severe adverse effects on the human body (18). This compound, a derivative of glucose, does not necessarily suppress the growth of cancer in animals with a single dose (17). However, a combined dose of 2-DG and anticancer agents, such as cisplatin, metformin and doxorubicin, causes extensive apoptotic cell death of cancer cells (19-21). This characteristic action of a combined dose of 2-DG and anticancer drugs was considered to be due to suppression of the glycolytic pathway and inhibition of the prosurvival pathway, including Akt (B kinase) $(22,23)$. Therefore, it will be interesting to study how a combined dose of Phx-3 and 2-DG may affect the growth of cancer cells.

Hepatocellular carcinoma, which is increasing worldwide (24) and is characterized by intra- and extrahepatic invasiveness (25), is resistant to anticancer agents. Agents to prevent the growth of hepatocellular cancer cells by inducing the apoptosis of these cancer cells are urgently required. Therefore, it is of interest to examine whether Phx-3 may exert anticancer activity against hepatocellular cancer cells, in a single or a combined dose with 2-DG, since there is no report on the cytotoxic and pro-apoptotic effects of $\mathrm{Phx}-3$ on hepatocellular cancer cells. Moreover, it will be important to investigate how a combined dose of Phx-3 and 2-DG may exert cytotoxic and pro-apoptotic effects against normal hepatocellular cells, in order to study the adverse effects of the combination of these compounds on the normal liver.

In this study, we investigated the effects of a single dose of Phx-3 or 2-DG and those of the combined use of Phx-3 and 2-DG on hepatocellular carcinoma cell lines, the rat dRL-84 and the human HepG2, as well as on the normal rat hepatocellular cell line RLN-10, in terms of the pro-apoptotic and cytotoxic effects in these cells.

\section{Materials and methods}

Reagents. Phx-3 was prepared by reacting $o$-aminophenol with bovine erythrocyte suspension, according to the method of Nakachi et al (26). The chemical structure of Phx-3 is depicted in Fig. 1. Phx-3 was dissolved in a mixture of dimethylsulfoxide (DMSO) and ethyl alcohol (3:1) as a vehicle to make a 20-mM solution. 2-Deoxy-D-glucose (2-DG) (Wako Pure Chemicals, Tokyo, Japan) was dissolved in distilled water to obtain a $1 \mathrm{M}$ stock solution, and was diluted with an incubation solution such as Dulbecco's modified Eagle's medium (DMEM) (Nissui Seiyaku Co., Ltd., Tokyo).

Cell lines and culture condition. The rat hepatocellular carcinoma cell line dRLh-84, the rat normal liver cell line RLN-10 and the human hepatocellular carcinoma cell line HepG2 which were obtained from the Health Science Research Resources Bank (Osaka, Japan), were cultured in DMEM supplemented with $10 \%$ fetal calf serum (FCS) (PAA Laboratories, GmbH, Austria), $2 \mathrm{mM}$ glutamine and $100 \mathrm{U} / \mathrm{ml}$ of penicillin. All of

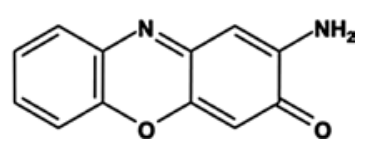

Figure 1. Chemical structure of 2-aminophenoxazine-3-one ( $\mathrm{Phx}-3)$.

the cells were cultured in a humidified incubator containing $5 \% \mathrm{CO}_{2} / 95 \%$ air at $37^{\circ} \mathrm{C}$.

Assessment of cell morphology. For morphological assessment, each of the three cell lines [the rat hepatocellular carcinoma cell line, dRLh-84, the rat normal liver cell line, RLN-10 and the human hepatocellular carcinoma cell line HepG2 $\left.\left(2 \times 10^{5}\right)\right]$ were incubated for $24 \mathrm{~h}$, with or without 2 or $10 \mu \mathrm{M}$ Phx-3 and/or $10 \mathrm{mM} 2-\mathrm{DG}$ in a 12 -well plate. The cells were then digested with trypsin, and a suspension of each cell line was sedimented and fixed on a slide glass, using a Shandon Cyotospin II (Shandon, Pittsburgh, PA, USA). The preparations were then stained with May-Grünwald-Giemsa solution and examined by a BZ-9000 digital microscope (Keyence Co., Osaka, Japan).

Estimation of viability of the hepatocellular carcinoma cell lines $d R L h-84$ and Hep 2 and the rat normal cell line RLN-10 treated with different concentrations of Phx-3 or 2-DG alone or in combination with 2-DG. The hepatocellular carcinoma cell lines dRLh-84 and HepG2 and the rat normal cell line RLN-10 $\left(5 \times 10^{3}\right)$ were incubated with or without various concentrations of $\mathrm{Phx}-3$ or $2-\mathrm{DG}$ for $48 \mathrm{~h}$ in 96-well plates. At 24 or $48 \mathrm{~h}$, a solution of CellTiter-Blue ${ }^{\mathrm{TM}}$ (Promega, Madison, WI) $(5 \mathrm{mg} / \mathrm{ml})$ was added to each well, and the cells were incubated for an additional $1.5 \mathrm{~h}$. The fluorescence was then recorded at $560 \mathrm{~nm}$ excitation/590 $\mathrm{nm}$ emission wavelengths.

Apoptosis dectection. Apoptotic cells were quantitatively evaluated by flow cytometry, using the Annexin-V-fluorescein staining kit (Wako Pure Chemicals). Each of the three cell lines dRLh-84, RLN-10 and HepG2 $\left(2 \times 10^{5}\right)$ were treated with $0,2.5$ or $10 \mu \mathrm{M}$ Phx-3 for $18 \mathrm{~h}$ with or without 2 or $10 \mathrm{mM}$ 2-DG. The cells were then harvested and washed once with phosphate-buffered saline (PBS, pH 7.4). An aliquot of $100 \mu \mathrm{l}$ of cell suspension was stained with $2 \mu \mathrm{l}$ of fluorescein isothiocyanate (FITC)-labeled Annexin-V (Annexin-V-FITC) and propidium iodide (PI). Samples were incubated for $15 \mathrm{~min}$ at room temperature, after which $1 \mathrm{ml}$ of binding buffer was added. The levels of fluorescent staining of the cells were analyzed using a flow cytometer (Partec PAS, Partec, Münster, Germany) to estimate the population of apoptotic cells at the early stage (Annexin-V positive and PI negative fractions) and the late apoptotic stage (Annexin-V positive and PI positive fractions), in each of three cell lines treated with different concentrations of Phx-3 with or without $10 \mathrm{mM}$ 2-DG.

Detection of loss of the mitochondrial membrane potential in hepatocellular carcinoma cells (dRLh-84-and HepG2) and rat normal hepatocellular cells $(R L N-10)$. The loss of mitochondrial membrane potential in the two hepatocellular carcinoma cell lines, dRLh-84-and HepG2, and the rat normal hepatocellular cell line, RLN-10, was examined by 
5,5',6,6'-tetrachloro-1,1',3,3'-tetraethylbenzimidazolylcarbocyanine iodide (JC-1). Briefly, the cells $\left(2 \times 10^{5}\right)$ were seeded in 12-well plates and were treated with $0,2,5$ or $10 \mu \mathrm{M} \mathrm{Phx}-3$ for $6 \mathrm{~h}$ with or without 2 or $10 \mathrm{mM}$ 2-DG. The cells were rinsed twice with PBS, then stained with $1 \mathrm{ml} 10 \%$ RPMI1640 medium containing $5 \mu \mathrm{mol} / 1 \mathrm{JC}-1$ (Molecular Probes, USA) for $30 \mathrm{~min}$ at $37^{\circ} \mathrm{C}$. The cells were then rinsed twice with ice-cold PBS, resuspended in 1-ml ice-cooled PBS, and instantly assessed for red and green fluorescence using a flow cytometer (Partec). JC-1 monomer green signals were detected at an emission wavelength of $535 \mathrm{~nm}$ (excitation wavelength $485 \mathrm{~nm}$ ) and the JC-1 aggregate red signals at an emission wavelength of $595 \mathrm{~nm}$ (excitation wavelength $540 \mathrm{~nm}$ ).

Immunoblotting analysis. Immunoblotting of LC3B and p62 in HepG2 cells was performed as previously described (27). HepG2 cells treated with $\mathrm{Phx}-3$ and/or 2-DG were lysed with RIPA lysis buffer (Santa Cruz Biotechnology Inc., Santa Cruz, CA) containing $1 \mathrm{mM}$ PMSF, $0.15 \mathrm{U} / \mathrm{ml}$ aprotinin, $10 \mathrm{mM}$ EDTA, $10 \mathrm{mg} / \mathrm{ml}$ sodium fluoride, and $2 \mathrm{mM}$ sodium orthovanadate. Cellular proteins were separated by SDS-PAGE and transferred onto Immobilon-P membranes (Millipore, Bedford, MA). The membranes were probed with the primary antibodies (Abs) anti-microtubule-associated protein 1 light chain 3 (LC3) B Ab (Novus Biologicals, Inc., Littleton, CO), anti-p62 Ab (Santa Cruz, CA), and anti-GAPDH monoclonal (m) Ab (Santa Cruz, CA). Immunoreactive proteins were detected with horseradish peroxidase-conjugated secondary Abs and an enhanced chemiluminescence reagent (ECL) (Millipore). Densitometry was performed using a Molecular Imager, ChemiDoc XRS System (Bio-Rad, Richmond, CA).

\section{Results}

First, we studied the cytotoxic effects of different concentrations of Phx-3 and 2-DG on the rat hepatocellular carcinoma cell line dRLh-84 (Fig. 2A), the human hepatocellular carcinoma cell line HepG2 (Fig. 2B) and the rat normal hepatocellular cell line RLN-10 (Fig. 2C), which were examined for $48 \mathrm{~h}$. As seen in Fig. 2A, a single dose of 2-DG $(0.5-20 \mathrm{mM})$ or Phx-3 (1-50 $\mu \mathrm{M})$ caused a dose-dependent decrease of viable dRLh- 84 cells at 24 and $48 \mathrm{~h}$. The number of viable cells was decreased to 60 and $40 \%$ of the control (without 2-DG) in the presence of $10 \mathrm{mM} 2-\mathrm{DG}$ at 24 and $48 \mathrm{~h}$, respectively (the upper columns in Fig. 2A), and the viable cells disappeared in the presence of $10 \mu \mathrm{M}$ or more Phx-3, at 24 and $48 \mathrm{~h}$, in dRLh-84 cells (the lower columns in Fig. 2A). The cytotoxic effects of the combined dose of Phx-3 (1-50 $\mu \mathrm{M})$ and $10 \mathrm{mM}$ 2-DG were additive in dRLh-84 cells (lower columns in Fig. 2A). Similar results were obtained for HepG2 cells (Fig. 2B) and the RLN-10 cells (Fig. 2C) treated with a single dose of 2-DG or Phx-3 or a combined dose of Phx-3 and 2-DG at different concentrations for $48 \mathrm{~h}$. The $\mathrm{IC}_{50}$ of $\mathrm{Phx}-3$ was estimated as $7.50 \pm 0.20 \mu \mathrm{M}$ for dRLh-84 cells, $6.58 \pm 0.61 \mu \mathrm{M}$ for HepG2 cells and $5.97 \pm 0.20 \mu \mathrm{M}$ for RLN-10 cells.

Next, we studied the morphological changes of the rat hepatocellular carcinoma cell line dRLh-84, the human hepatocellular carcinoma cell line HepG2 and the rat normal hepatocellular cell line RLN-10 with or without Phx-3 (2 or $10 \mu \mathrm{M})$ and/or 2-DG (10 mM) (Fig. 3). There were a few apoptotic features, including a condensed nucleus and cell shrinkage, in the control dRLh-84 cells without Phx-3 and 2-DG treatment (the upper left two panels in Fig. 3A), or in dRLh-84 cells with $10 \mathrm{mM} 2-\mathrm{DG}$ alone (the upper right two panels in Fig. 3A). However, in the latter case, numerous valuoles possibly associated with autophagy were observed in the cytosol of dRLh-84 cells treated with $10 \mathrm{mM} 2-\mathrm{DG}$ alone. When $2 \mu \mathrm{M} \mathrm{Phx}-3$ was added to the dRLh- 84 cells, the number of apoptotic cells with condensed nucleus and cell shrinkage increased significantly (the lower left two panels), and included many vacuoles in the cytosol, suggesting that apoptosis and autophagy are proceeding simultaneously. The number of cells with extremely condensed nuclei and cell shrinkage increased in dRLh-84 cells treated with $2 \mu \mathrm{M} \mathrm{Phx}-3$ and $10 \mathrm{mM}$ 2-DG (the lower right two panels in Fig. 3A), indicating that almost every cell underwent the apoptotic cell death.

Similar morphological changes were observed in HepG2 cells (Fig. 3B). Specifically, few apoptotic cells were seen in HepG2 cells treated with $10 \mathrm{mM}$ 2-DG alone, but vacuole formation was observed in the cytosol of the cells (the upper right two panels). After exposure of the cells to $10 \mu \mathrm{M}$ Phx-3 alone, the HepG2 cells experienced apoptosis, accompanied by vacuole formation in the cells (the lower left two panels). When $10 \mu \mathrm{M}$ Phx-3 and $10 \mathrm{mM}$ 2-DG were administered to HepG2 cells, the viable cells decreased and the remaining cells exhibited typical apoptotic morphology with many cytosolic valuoles (the lower right two panels in Fig. 3B). This observation was further examined, as described later in connection with Fig. 6.

When the rat normal hepatocellular cell line RLN-10 was singly treated with 2-DG $(10 \mathrm{mM})$, there was no significant apoptotic features or cytosolic vacuole formation (the upper right two panels in Fig. 3C). However, apoptotic morphology was observed when cells were treated singly with Phx-3 $(2 \mu \mathrm{M})$, but minimal vacuole formation was observed in this case. When RLN-10 cells were treated with $2 \mu \mathrm{M}$ Phx-3 and $10 \mathrm{mM}$ 2-DG, the viable cells decreased and the remaining cells exhibited typical apoptotic morphology, but with minimal vacuole formation (the lower right two panels in Fig. 3C), suggesting that the combined dose of Phx-3 and 2-DG induced apoptotic cell death in RLN-10 cells without causing autophagy.

In order to confirm apoptotic cell death in the three afore-mentioned hepatocellular cell lines, we examined the population of apoptotic cells in these cell lines when treated with Phx-3 or/and 2-DG for 18 h (Fig. 4). Fig. 4A presents representative data obtained by flow cytometry for the population of apoptotic cells in dRLh-84 cells, which were treated with Phx-3 alone (0, 2, 5 and $10 \mu \mathrm{M}), 2-\mathrm{DG}$ alone $(0,2$ and $10 \mathrm{mM}$ ), or with both Phx-3 (2, 5 and $10 \mu \mathrm{M})$ and 2-DG (2 or $10 \mathrm{mM}$ ) for $18 \mathrm{~h}$. Subsequently, it was found that the population of late stage apoptotic cells [Annexin- $\mathrm{V}(+)$ and $\mathrm{PI}(+)$ ] increased up to $90.5,92.8$ and $91.5 \%$ in dRLh-84 cells treated with a single dose of 2,5 and $10 \mu \mathrm{M} \mathrm{Phx}-3$, respectively. The population of late stage apoptotic cells increased up to $88,94.5$ and $95.4 \%$ in the presence of 2,5 and $10 \mu \mathrm{M} \mathrm{Phx}-3$ in combination with $2 \mathrm{mM} 2-\mathrm{DG}$, and up to $92.7,93.5$, and $92 \%$ in combination with $10 \mathrm{mM}$ 2-DG (these data are depicted as histograms in Fig. 4B). In these cases, the population of early apoptotic cells [Annexin-V(+) and PI(-)] and necrotic cells [Annexin-V(-) 
A
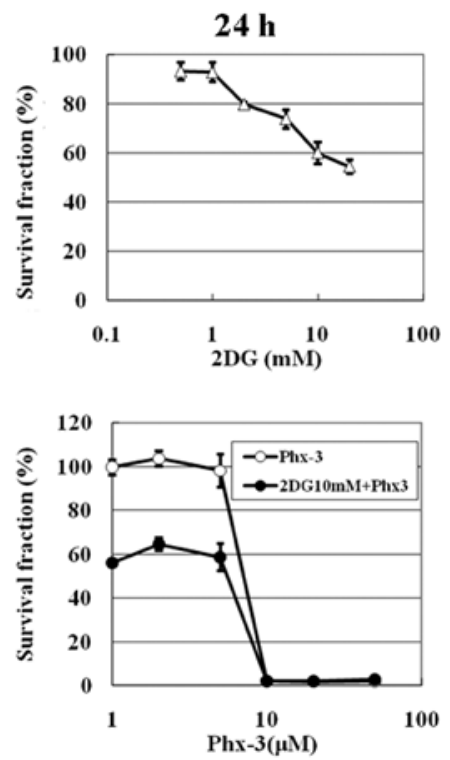

B
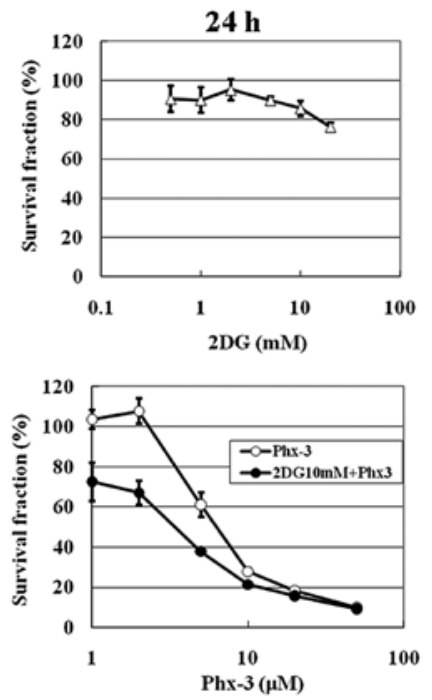

C
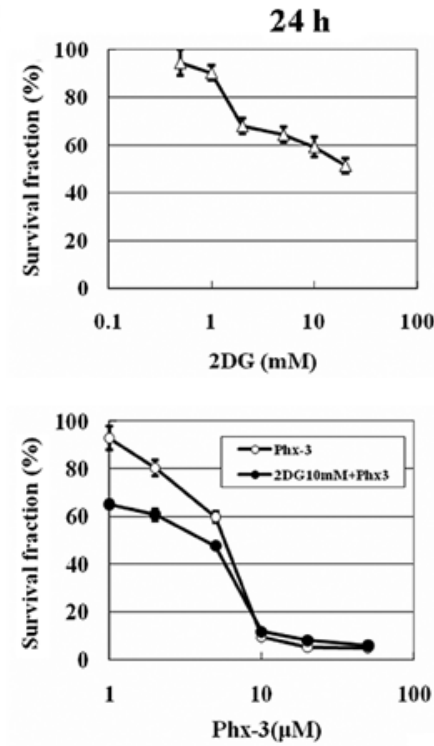
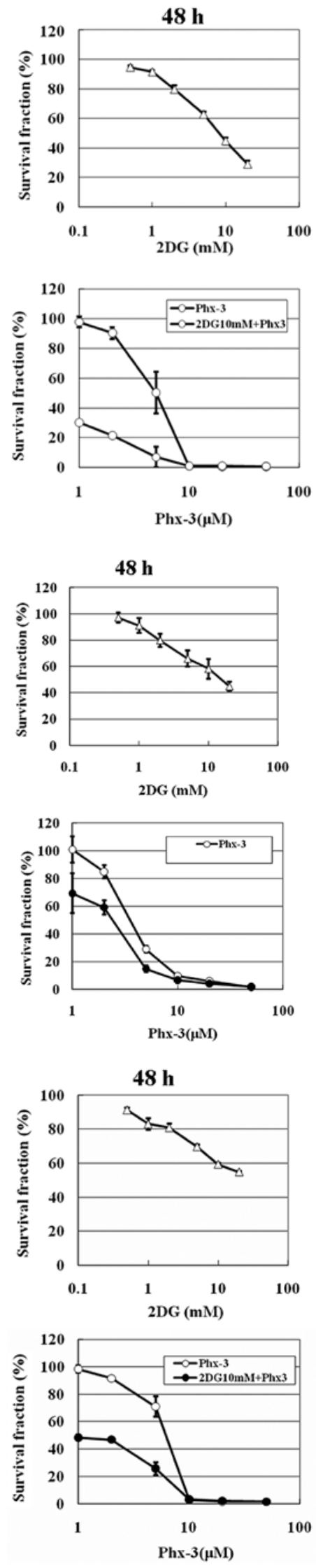

Figure 2. Cytotoxic effects of a single dose of Phx-3 or 2-DG and a combined dose of Phx-3 and 2-DG on the rat hepatocellular carcinoma cell line dRLh-84, the human hepatocellular carcinoma cell line HepG2, and the rat normal liver cell line RLN-10. Each of the three cell lines was treated with a single dose of Phx-3 (1-50 $\mu \mathrm{M})$ or 2-DG $(0.5-20 \mathrm{mM})$ or a combined dose of Phx-3 (1-50 $\mu \mathrm{M})$ and 2-DG (10 mM), and the number of viable cells (as a percentage of the control) was estimated fluorometrically by using the CellTiter-Blue ${ }^{\mathrm{TM}}$ method. Open triangles, 0.5-20 mM 2-DG; open circles, 1-50 $\mu \mathrm{M}$ Phx-3; closed circles, $10 \mathrm{mM}$ 2-DG plus 1-50 $\mu \mathrm{M}$ Phx-3. (A) Rat hepatocellular carcinoma cell line dRLh-84; (B) human hepatocellular carcinoma cell line HepG2; (C) rat normal liver cell line RLN-10. 

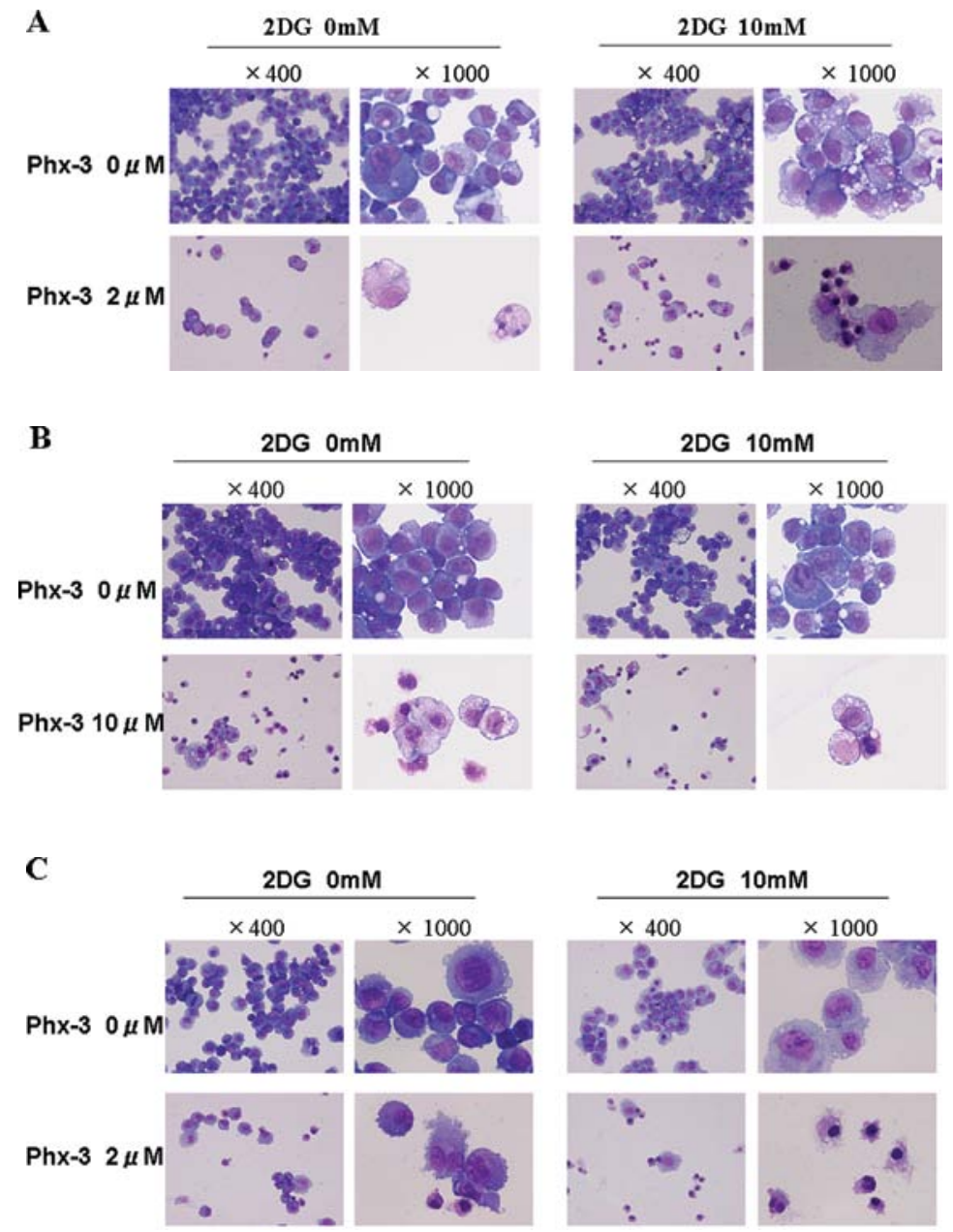

Figure 3. Morphological changes in the rat hepatocellular carcinoma cell line dRLh-84, the human hepatocellular carcinoma cell line HepG2 and the rat normal liver cell line RLN-10 treated with a single dose of 2-DG, Phx-3 or a combined dose of 2-DG and Phx-3. The left four panels in each figure depict cells treated without 2-DG and Phx-3 or with 2 or $10 \mu \mathrm{M} \mathrm{Phx}-3$ alone; the right four panels in each figure depict cells treated with $10 \mathrm{mM} 2-\mathrm{DG}$ alone or $10 \mathrm{mM}$ 2-DG plus 2 or $10 \mu \mathrm{M}$ Phx-3. Magnification, x400 or x1000. Morphology of (A) the rat hepatocellular carcinoma cell line dRLh-84; (B) the human hepatocellular carcinoma cell line, HepG2; (C) the rat normal liver cell line, RLN-10.

and PI (+)] was negligible. These results indicate that almost all dRLh-84 cells experienced late stage apoptotic cell death following treatment with $2 \mu \mathrm{M}$ or more $\mathrm{Phx}-3$ for $18 \mathrm{~h}$, with or without 2-DG.

The histograms in Fig. 4B-D reflect the population of late stage apoptotic cells in dRLh-84, HepG2 and RLN-10 cells treated with Phx-3 or 2-DG or a combined dose of Phx-3 and 2-DG for $18 \mathrm{~h}$, which was analyzed by flow cytometry. It is evident in Fig. 4C that a single dose of Phx-3 (2-10 $\mu \mathrm{M})$ and a combined dose of Phx-3 (2-10 $\mu \mathrm{M})$ and 2-DG (2 or $10 \mathrm{mM})$ greatly increased the population of late apoptotic cells, while 2-DG alone caused an increase in late apoptotic cells in the human hepatocellular carcinoma cell line HepG2, dependent on the dose of Phx-3. For a combined dose of $2 \mu \mathrm{M}$ Phx-3 and $10 \mathrm{mM}$ 2-DG, Phx-3 caused pro-apoptotic effects on HepG2 cells in an additive manner, because the difference (20\%) between the apoptotic cells in HepG 2 cells treated with $10 \mathrm{mM}$ 2-DG and $2 \mu \mathrm{M} \mathrm{Phx}-3$ (apoptosis fraction, 80\%) and those treated with $10 \mathrm{mM} 2-\mathrm{DG}$ and $0 \mu \mathrm{M} \mathrm{Phx}-3$ (apoptosis fraction, $60 \%$ ) is comparable to the difference $(21 \%)$ between the apoptotic cells in HepG2 cells treated with 0 mM 2-DG and $2 \mu \mathrm{M}$ Phx-3 (apoptosis fraction, 39\%) and those treated with $0 \mathrm{mM}$ 2-DG and $0 \mu \mathrm{M} \mathrm{Phx}-3$ (apoptosis fraction, 18\%) (Fig. 4C).

In contrast, a combined dose of $2 \mu \mathrm{M}$ Phx-3 with $10 \mathrm{mM}$ 2-DG exerted synergistic pro-apoptotic effects in the rat normal hepatocellular cell line RLN-10, because the apoptotic cells increased up to $73 \%$ in the RLN-10 cells treated with $2 \mu \mathrm{M}$ Phx-3 and $10 \mathrm{mM}$ 2-DG, while $2 \mu \mathrm{M}$ Phx-3 or $10 \mathrm{mM}$ $2-\mathrm{DG}$ alone did not increase the population of apoptotic RLN-10 cells (Fig. 4D).

Since it has been recognized that depolarization of the mitochondria is induced prior to the apoptotic events in cancer cells treated with anticancer agents $(28,29)$ and $\mathrm{Phx}-3$ $(14,30,31)$, we studied the effect of Phx-3 and 2-DG on the depolarization of the mitochondria in the hepatocellular carcinoma cell lines, dRLh-84 and HepG2, the and normal cell line, RLN-10. Fig. 5A presents the effect of $\mathrm{Phx}-3$ (0, 2 or $5 \mu \mathrm{M}$ ) on depolarization of the mitochondria in the rat hepatocellular carcinoma dRLh-84 cells, analyzed by flow cytometry. Consequently, significant depolarization of the mitochondria in dRLh-84 was caused by $5 \mu \mathrm{M}$ Phx-3. The histograms in Fig. 5B-D demonstrate depolarization of the mitochondria in dRLh- 84 cells, HepG 2 cells and RLN-10 cells 
$\mathbf{A}$

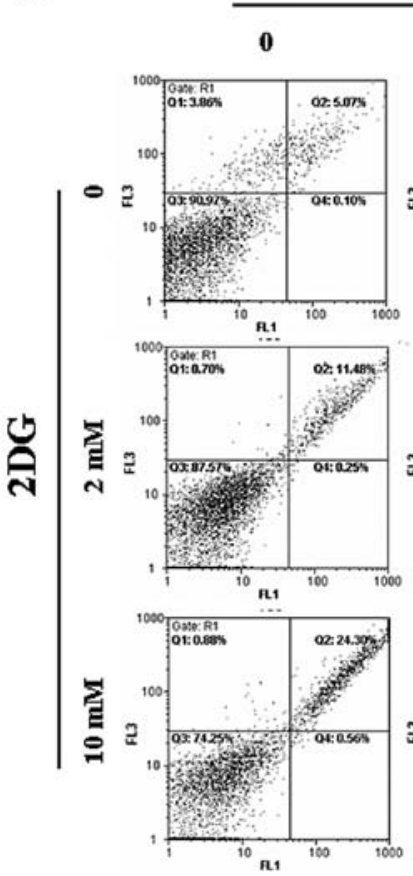

Phx-3
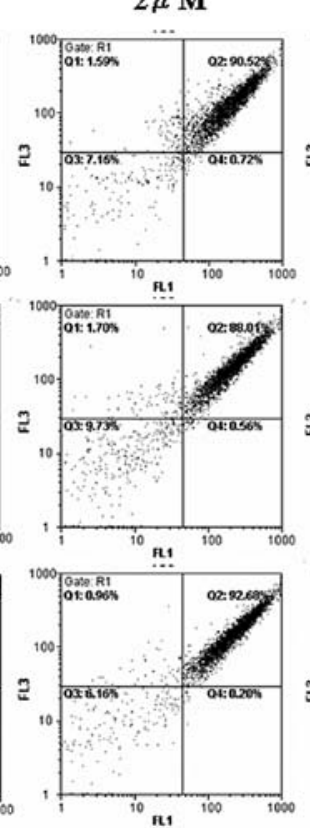
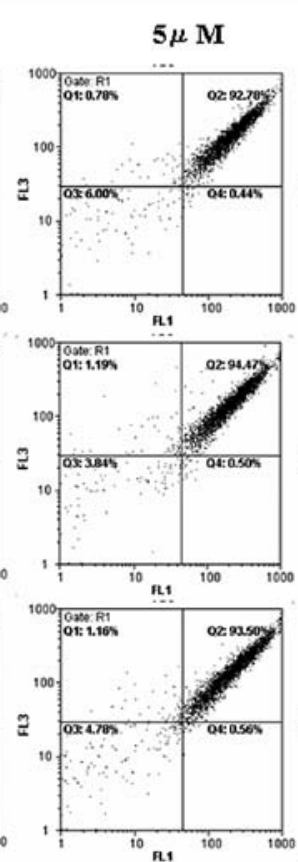

$10 \mu \mathrm{M}$
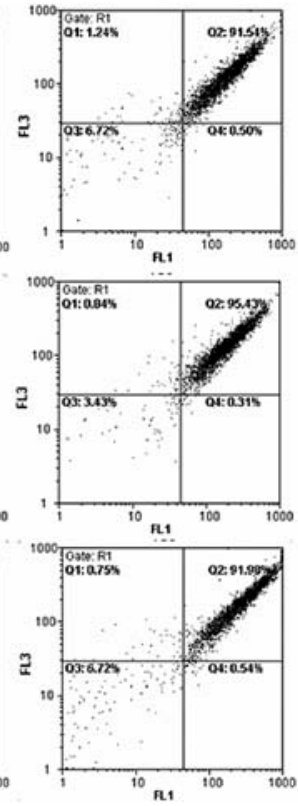

B

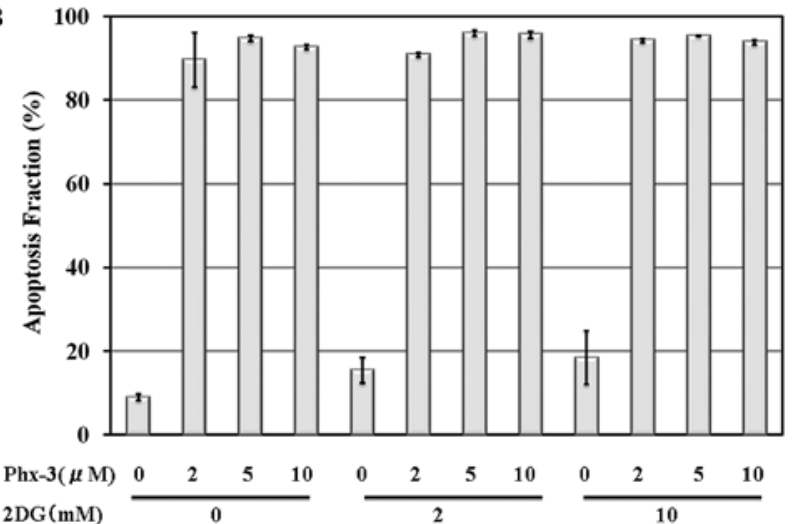

D

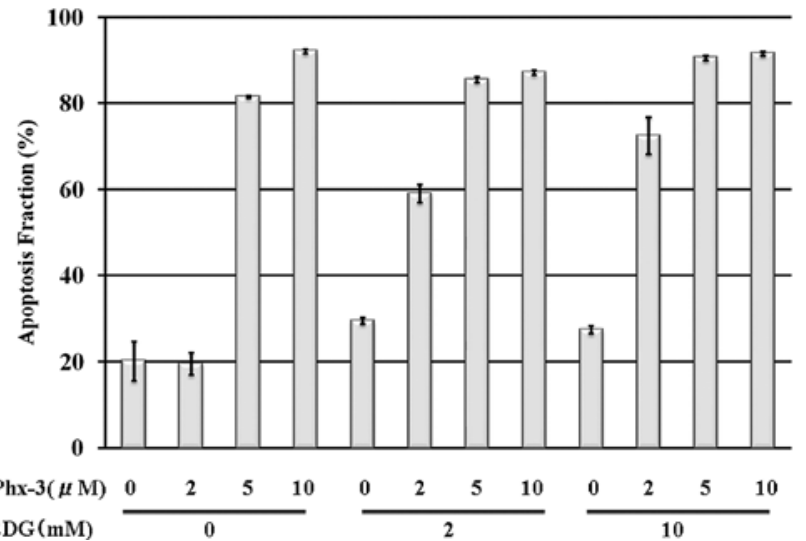

with different concentrations of Phx-3 and/or 2-DG. It is noteworthy that depolarization of the mitochondria was induced in hepatocellular carcinoma cells including dRLh-84 cells and HepG2 cells, dependent on the dose of Phx-3, though 2-DG did not cause depolarization of the mitochondria in these cells (Fig. 5B and C), and that depolarization of the mitochondria was not induced in the rat normal RLN-10 cells in the presence

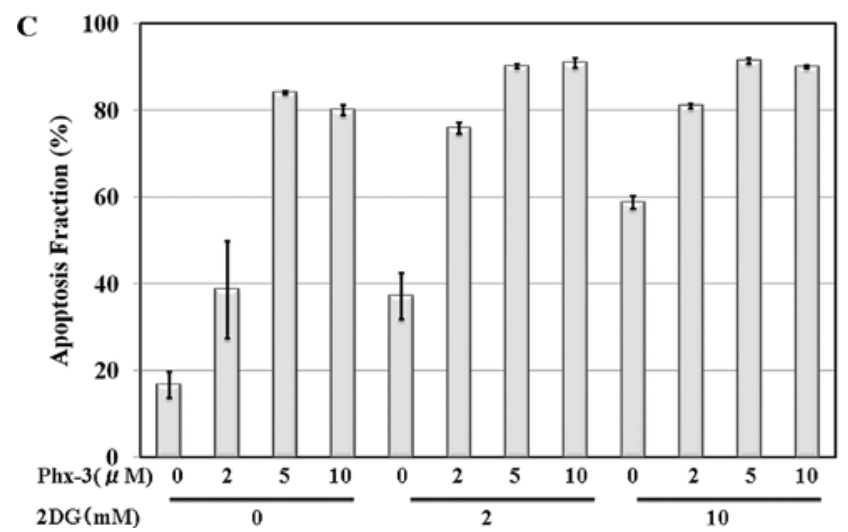

Figure 4. Flow cytometric analysis of the population of apoptotic cells with early stage apoptosis, late stage apoptosis/necrosis and necrosis in the rat hepatocellular carcinoma cell line dRLh-84, human hepatocellular carcinoma cell line HepG2 anthe rat normal liver cell line RLN-10, as detected by Annexin-V and PI staining. (A) dRLh-84 cells, rat hepatocellular carcinoma cell line dRLh-84 was grown with or without Phx-3 and/or 2-DG for $18 \mathrm{~h}$ The cells were stained with FITC-Annexin-V and PI, and then examined by flow cytometry. In each dot plot, the percentages of Annexin-V(-), PI (-) (viable cells, bottom-left quadrant), Annexin- $\mathrm{V}(+) \mathrm{PI}(-)$ (cells in early stage apoptosis, bottom-right quadrant), Annexin- $\mathrm{V}(+) \mathrm{PI}(+)$ (cells in late stage apoptosis, top-right quadrant), and Annexin-V(-)PI(+) (cells in necrosis, topleft quadrant) are detected. (B) Histograms of the apoptotic cell population of rat hepatocellular carcinoma cell line dRLh-84 cells. (C) Histograms of the apoptotic cell population of human hepatocellular carcinoma cell line HepG2 cells. (D) Histograms of the apoptotic cell population of rat normal liver cell line RLN-10.

of various concentrations of $\mathrm{Phx}-3$ and/or 2-DG, compared with the control without Phx-3 and 2-DG (Fig. 5D).

As seen in Fig. 3A and B, a significant increase of cytosolic vacuoles was observed in the hepatocarcinoma cell lines, dRLh-84 and HepG 2 treated with 2 or $10 \mu \mathrm{M}$ Phx-3. In addition, combined treatment with $\mathrm{Phx}-3$ and 2-DG appeared to enhance the number of cytosolic vacuoles in these hepatocar- 
A $\quad$ Phx-3

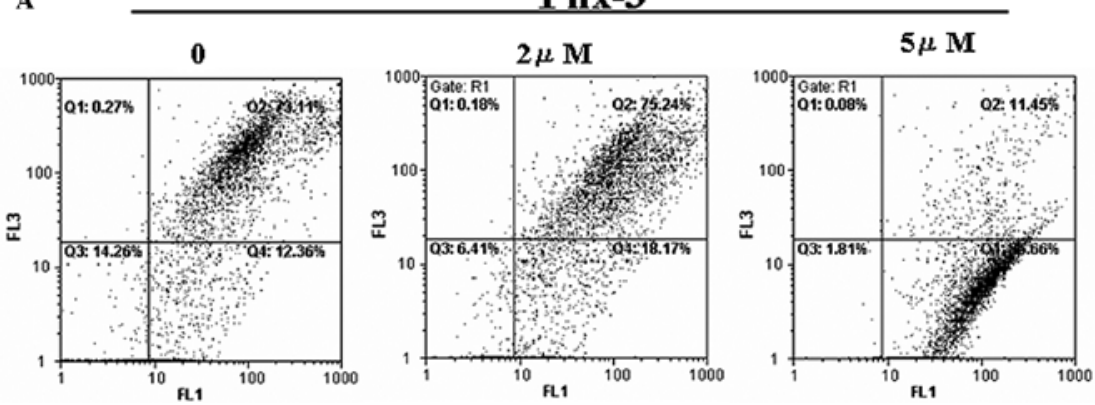

B

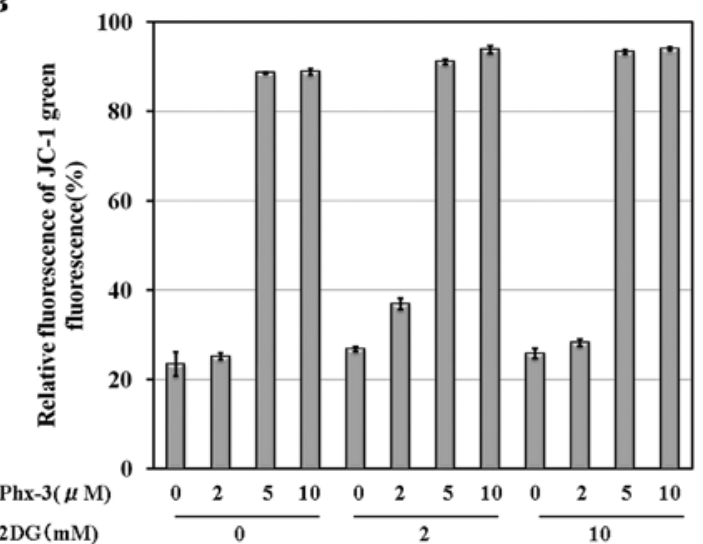

D

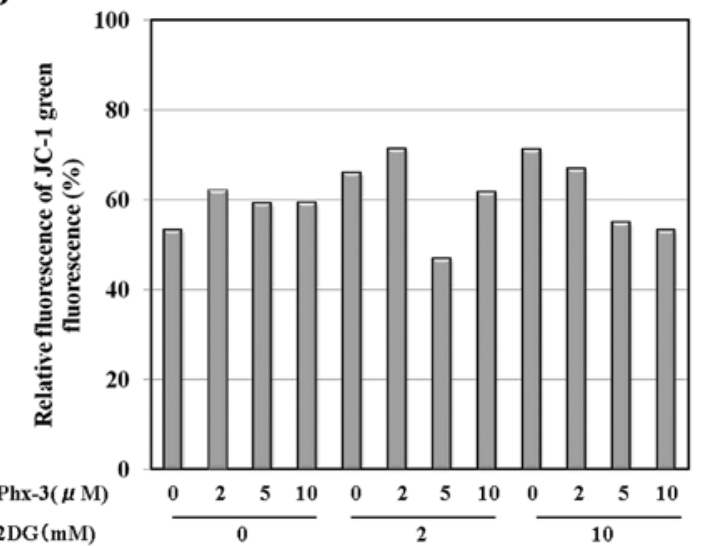

C

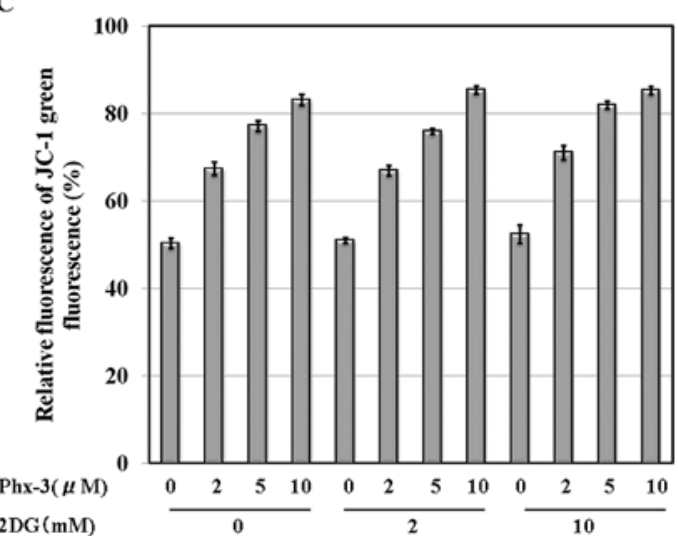

Figure 5. Depolarization of the mitochondria in rat hepatocellular carcinoma cell line dRLh-84, human hepatocellular carcinoma cell line HepG2 and rat normal liver cell line RLN-10 treated with or without Phx-3 and 2-DG. (A) Flow cytometric analysis of the depolarized mitochondria in dRLh-84 cells treated with or without 2 or $5 \mu \mathrm{M} \mathrm{Phx}-3$ for $6 \mathrm{~h}$. Population of cells with the depolarized mitochondria in (B) dRLh-84 cells treated with or without 2 or $10 \mathrm{mM} 2-\mathrm{DG}$ or 2,5 or $10 \mu \mathrm{M} \mathrm{Phx}-3$. (C) HepG2 cells treated with or without 2 or $10 \mathrm{mM} 2$-DG or 2,5 or $10 \mu \mathrm{M}$ Phx-3 or (D) in RLN-10 cells treated with or without 2 or $10 \mathrm{mM} 2-\mathrm{DG}$ or 2,5 or $10 \mu \mathrm{M} \mathrm{Phx}-3$.

cinoma cells (Fig. 3A and B), suggesting that autophagy might be induced in response to both 2-DG and Phx-3 in dRLh-84 and HepG 2 cells. Thus, we performed immunoblotting using antiLC3B and anti-p62 antibodies, in order to investigate whether or not autophagy was induced in HepG2 cells after treatment with Phx-3 and/or 2-DG. As shown in Fig. 6, an increased ratio of LC3B-II/LC3B-I (5.06) as well as a decreased p62 protein level was observed after 16-h exposure of HepG2 cells to Phx-3. This indicated that autophagy was induced in HepG2 cells in response to $\mathrm{Phx}-3$. 2-DG also induced autophagy but to a much lesser extent than Phx-3. Since combined treatment with $\mathrm{Phx}-3$ plus 2 -DG exhibited potent cytotoxicity against HepG2 cells (Fig. 3B), making it difficult to obtain a sufficient amount of protein from the cells, enhanced autophagy induction due to the combination of 2-DG and Phx-3 was not evident as seen in Fig. 6. However, these data suggest that autophagy was involved during cytotoxic induction in response to $\mathrm{Phx}-3$ and 2-DG.

\section{Discussion}

Phx-3 has been shown to exert strong anticancer activity against a variety of cancer cell lines in vitro and in vivo (12-15,30-34). However, the anticancer activity of this oxidative phenoxazine against hepatocellular carcinoma cells has not been investigated, though this kind of malignant tumor is intractable to chemotherapy, and its incidence is increasing worldwide $(24,25)$. We found in the present study that $10 \mu \mathrm{M}$ or more $\mathrm{Phx}-3$ exerted strong cytotoxic effects on the rat hepatocellular carcinoma cell line dRLh-84 and the human hepatocellular carcinoma cell line HepG2 with or without $10 \mathrm{mM}$ 2-DG during 24- or 48-h incubation (Fig. 2A and B). In addition, morphological (Fig. 3A and B) and flow cytometric analysis (Fig. 4B and C) revealed that treatment of dRLh-84 and HepG2 cells with a single dose of Phx-3 higher than $2 \mu \mathrm{M}$, induced apoptotic cell death. These results indicate that $\mathrm{Phx}-3$ is capable of exerting strong anticancer activity against 


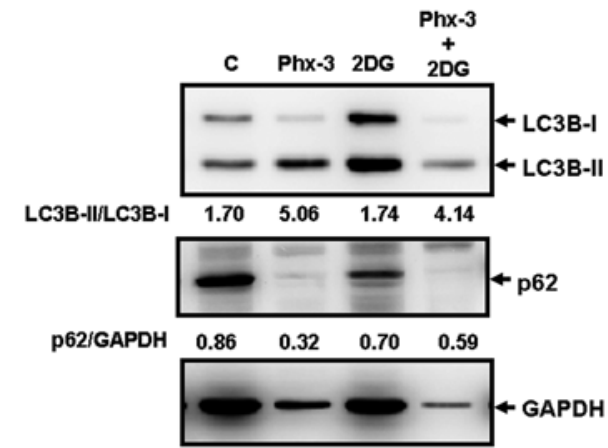

Figure 6. Immunoblotting patterns of LC3B-I, LC3B-II and p62 in HepG2 cells treated with Phx-3 and/or 2-DG. HepG2 cells were treated with either Phx-3 $(5 \mu \mathrm{M})$ and/or 2-DG (10 mM) for $16 \mathrm{~h}$. After lysis of the cells, cellular proteins were separated by 15\% SDS-PAGE for LC3B and $11.25 \%$ SDS-PAGE for $\mathrm{p} 62$ proteins, and thereafter immunoblotted with anti-LC3B $\mathrm{Ab}$ and anti-p62 $\mathrm{Ab}$, respectively. Immunoblotting with anti-GAPDH $\mathrm{mAb}$ was performed as an internal control. An increased ratio of LC3B-II/LC3B-I along with a decreased p62 protein level indicates autophagy induction in response to both Phx-3 and 2-DG.

hepatocellular carcinoma cells in vitro. The mechanism for the anticancer activity of Phx-3 in hepatocellular carcinoma cells may be explained primarily by depolarization of the mitochondria in cells treated with this phenoxazine, as demonstrated in Fig. 5B and C, and in recent reports $(30,31)$. This view is also supported, by the fact that depolarization of the mitochondria has been recognized as an important change in the final stage of apoptotic events in cancer cells $(28,29)$.

We found that 2-DG, an inhibitor of hexokinase that suppresses the glycolytic metabolism and thereby decreases ATP levels in cancer cells (16), does not necessarily exert strong anticancer activity against the hepatocarcinoma cell lines dRLh-84 and HepG2, because a small increase (moderate increase) of the apoptotic cells was indicated with 2-DG alone in dRLh-84 (Fig. 4B) or HepG2 (Fig. 4C). The minimal or moderate anticancer activity of 2-DG on these hepatocarcinoma cells may be associated with autophagy induction by 2-DG, which has been considered as an important mechanism of resistance to cellular stress such as deficiency of ATP $(22,23)$. This view will be supported by our present results that significant cytosolic vacuole formation, which is a hallmark of the autophagic cells, was present in dRLh-84 cells and HepG2 cells treated with 2-DG (Fig. 2A and B) and that the ratio of LC3B-II/LC3B-I (1.74) was slightly increased and the p62 protein level (0.7) was decreased in HepG2 cells exposed to 2-DG for $16 \mathrm{~h}$ (Fig. 6).

Phx-3 seemed to cause both apoptosis and autophagy in the hepatocarcinoma cell lines dRLh-84 and HepG2 (Fig. 2A and B, Figs. 3 and 6). The autophagy accompanying cytosolic vacuole formation in these cells induced by $\mathrm{Phx}-3$ may be explained by the decrease in pHi in these cells which inhibits the glycolytic pathway and decreases ATP in the cells, as has been suggested by recent reports $(14,15)$. The apoptosis in the cells treated with Phx-3 may be due to depolarization of the mitochondria, as described above. It is noteworthy that when Phx-3 was administered to dRLh- 84 cells and HepG 2 cells, in combination with 2-DG, the population of apoptotic cells increased greatly and additively in these cells (Fig. 4B and C). Such additive anticancer effects of Phx-3 plus 2-DG suggest the coordination of these compounds in promoting mitochondrial depolarization by $\mathrm{Phx}-3$ (Fig. 5B and C) and in activating AMP-activated protein kinase (AMPK) by 2-DG $(22,23)$. This result is consistent with the reports (19-21) that 2-DG exerts additive or synergistic anticancer effects when administered to several cancer cells, in combination with various anticancer drugs, including cisplatin and metformin.

The pro-apoptotic effects of a combined dose of Phx-3 and 2-DG in the normal rat cell line RLN-10 were extensive and synergistic (Fig. 4D). Tamura et al (35) indicated that anticancer agents such as cisplatin, doxorubicin and mitomycin prevent growth of rat hepatocytes from hepatectomized rats, and suggested that systemic chemotherapy would be disturbed by the impairment of the liver. Therefore, our present finding may suggest the possibility that a combined dose of Phx-3 and 2-DG, which caused extensive cytotoxic and pro-apoptotic effects on a rat normal hepatocelluar cell line RLN-10, may have adverse effects on the liver when used in vivo, though this view should be confirmed by further examination.

Depolarization of the mitochondria has been recognized as an important change in the final stage of apoptotic evens in cancer cells $(28,29)$. We found that significant depolarization of the mitochondria was induced in dRLh-84 and HepG2 cells treated with $2 \mu \mathrm{M}$ or more Phx-3 for $18 \mathrm{~h}$, without being affected by the presence of 2-DG (Fig. 5B and C). In spite of the strong action of Phx-3 on depolarizing the mitochondria in hepatocarcinoma cell lines, Phx-3 did not depolarize the mitochondria in the normal rat cell line RLN-10, with or without 2-DG (Fig. 5D). Such differences in the sensitivity of cancer cells and normal cells to $\mathrm{Phx}-3$ have been indicated by Che et al (14). They demonstrated that Phx-3 caused depolarization of the mitochondria in a variety of cancer cells from different organs, but has not this effect in normal cells, including human umbilical vein endothelial cells and HEL fibroblast cells. It is unclear how this difference in the sensitivity of the mitochondria to depolarization due to $\mathrm{Phx}-3$ between cancer cells and normal cells comes about. There may be differences in the nature of the mitochondria between these cells, or some different mechanisms for regulating the mitochondrial depolarization between these cells. However, such a difference would be advantageous for the therapeutic strategy of selectively causing apoptosis in cancer cells.

In conclusion, Phx-3 itself was found to have cytotoxic and pro-apoptotic activity against hepatocellular carcinoma cells, and it exerted much stronger anticancer activity against these cells when they were treated with Phx-3 and 2-DG together. However, the adverse effects of a combined dose of Phx-3 and 2-DG should be considered for chemotherapeutic application to liver carcinoma, because the viability of rat normal hepatocellular cells was greatly affected by a combined dose of these compounds.

\section{Acknowledgements}

The present study was supported by funds from the High-Tech Research Project for Private Universities and a matching fund subsidy from the Ministry of Education, Culture, Sports and Technology, Japan (2008-2013), and by aGrant-in-Aid for Young Scientists (B) from the Ministry of Education, Culture, Sports and Technology, Japan (2009-2010) (no. 21790324). 


\section{References}

1. Warburg O: On the origin of cancer cells. Science 24: 309-314 1956.

2. Pelicano H, Martin DS, Xu RH and Huang P: Glycolysis inhibition for anticancer treatment. Oncogene 25: 4633-4646, 2006.

3. Xu RH, Pelicano H, Zhou Y, Carew JS, Feng L, Bhalla KN, Keating MI and Huang P: Inhibition of glycolysis in cancer cells: a novel strategy to overcome drug resistance associated with mitochondrial respiratory defect and hypoxia. Cancer Res 65: 613-621, 2005

4. Zhang F and An RI: Chemosensitizing and cytotoxic effects of 2-deoxy-D-glucose on breast cancer cells. J Cancer Res Ther 5: S41-S43, 2011.

5. Minakami S and Yoshikawa H: The effect of active cation transport, $\mathrm{pH}$ and inorganic phosphate concentration on erythrocyte glycolysis. J Biochem 59: 145-150, 1966.

6. Ui M: A role of phosphofructokinase in $\mathrm{pH}$ dependent regulation of glycolysis. Biochim Biophys Acta 124: 310-322, 1966.

7. Maidorn RP, Cragoe EJ and Tannock IF: Therapeutic intracellular $\mathrm{pH}$ as a possible mechanism of tumor selective therapy. $\mathrm{Br} \mathrm{J}$ Cancer 67: 297-303, 1993.

8. Izumi H, Torigoe T, Ishguchi H, Uramoto H, Yoshida Y, Tanabe M, Ise T, Murakmi T, Yoshida T, Nomoto M and Kohno K: Cellular $\mathrm{pH}$ regulators: potential promising molecular target for cancer chemotherapy. Cancer Treat Rev 29: 541-549, 2003.

9. Harguindey S, Arranz JL, Wahl ML, Orive G and Reshkin SJ: Proton transport inhibitors as potentially selective anticancer drugs. Anticancer Res 29: 2127-2136, 2009.

10. Rich IN, Worthington-White D, Garden OA and Musk P Apoptosis of leukemic cells accompanies reduction in intracellular $\mathrm{pH}$ after targeted inhibition of the $\mathrm{Na}^{+} / \mathrm{H}^{+}$exchanger. Blood 95: 1427-1434, 2000.

11. Kim KM and Lee YJ: Role of HER-2/neu signaling in sensitivity to tumor necrosis factor-related apoptosis-inducing ligand enhancement of TRAIL-mediated apoptosis by amiloride. J Cell Biochem 96: 376-389, 2005.

12. Che XF, Akiyama S and Tomoda A: Suppression of the proliferation of cancer cell lines, KB-3-1 and K562 cells preceded by a decrease in intracellular $\mathrm{pH}$ caused by phenoxazine derivatives. Oncol Rep 19: 1253-1258, 2008.

13. Zheng CL, Che XF, Akiyama S, Miyazawa K and Tomoda A: 2-Aminophenoxazine-3-one induces cellular apoptosis by causing rapid intracellular acidification and generating reactive oxygen species in human lung adenocarcinoma cells. Int J Oncol 36: 641-650, 2010 .

14. Che XF, Zheng CL, Akiyama $S$ and Tomoda $A$ 2-Aminophenoxazine-3-one and 2-amino-4,4a-dihydro4a,7-dimethyl-3H-phenoxazine-3-one cause cellular apoptosis by reducing higher intracellular $\mathrm{pH}$ in cancer cells. Proc Jpn Acad Ser B Phys Biol Sci 87: 199-213, 2011.

15. Nagata H, Che XF, Miyazawa K, Tomoda A, Konishi M, Ubukata $\mathrm{H}$ and Tabuchi T: Rapid decrease of intracellular $\mathrm{pH}$ associated with inhibition of $\mathrm{Na}^{+} / \mathrm{H}^{+}$exchanger precedes apoptotic events in the MNK45 and MNK74 gastric cancer cell lines treated with 2-aminophenoxazine-3-one. Oncol Rep 25 341-346, 2011

16. Parniak M and Kalant N: Incorporation of glucose into glycogen in primary cultures of rate hepatocytes. Can J Biochem Cell Biol 63: 333-340, 1985

17. Maschek G, Savaraj N, Priebe W, Braunshweiger P, Hamilton K, Tidmarsh GF, De Yong IR and Lampidis TI: 2-Deoxy-D-glucose increases the efficacy of adriamycin and paclitaxel in human osteosarcoma and non-small cell lung cancers in vivo. Cancer Res 64: 31-34, 2004

18. Singh D, Banerji AK, Dwarakanath BS, Tripathi RP, Gupta JP, Mathew TL, Ravindranath T and Jain V: Optimizing cancer radiotherapy with 2-deoxy-D-glucose. Strahlenther Onkol 181: 507-514, 2005

19. Simons AL, Ahmad IM, Mattson DM, Dornfeld KJ and Spitz DR: 2-Deoxy-D-glucose combined with cisplatin enhances cytotoxicity via metabolic oxidative stress in human head and neck cancer cells. Cancer Res 67: 3364-3370, 2007.
20. Sahra IB, Laurent K, Giuliano S, Larbret F, Ponzio G, Gounon P, Marchand-Brustel Y, Giorgetti-Peraldi S, Cormont $M$, Bertolotto C, Deckert M, Auberger P, Tanti JF and Bost F: Targeting cancer cell metabolism: the combination of metformin and 2-deoxyglucose induces $\mathrm{p} 3$-dependent apoptosis in prostate cancer cells. Cancer Res 70: 2465-2475, 2010.

21. Loar P, Wahl H, Kshirsagar M, Gossner G, Griffith K and Liu JR: Inhibition of glycolysis enhances cisplatin-induced apoptosis in ovarian cancer cells. Am J Obstet Gynecol 202: 371.e1-e8. 2010.

22. Zhong D, Xiong L, Liu T, Liu X, Liu X, Chen J, Sn S-Y, Khuri FR, Zong Y, Zhou Q and Zhou W: The glycolytic inhibitor 2-deoxyglucose activates multiple prosurvival pathways through IGFIR. J Biol Chem 284: 23225-23233, 2009.

23. Shara IB, Tanti JF and Bust F: The combination of metformin and 2-deoxyglucose inhibits autophagy and induces AMPKdependent apoptosis in prostate cancer cells. Autophagy 6: 670-671, 2010.

24. Schafer DF and Sorrell MF: Hepatocellular carcinoma. Lancet 353: 1253-1257, 1999.

25. Simonetti RG, Liberati A, Angiolini C and Pagliaro L: Treatment of hepatocellular carcinoma: a systemic review of randomized controlled trials. Ann Oncol 8: 117-136, 1997.

26. Nakachi T, Tabuchi T, Takasaki A, Arai S, Miyazawa K and Tomoda A: Anticancer activity of phenoxazines produced by bovine erythrocytes on colon cancer cells. Oncol Rep 23: $1517-1522,2010$.

27. Kawaguchi T, Miyazawa K, Moriya S, Ohtomo T, Che XF, Naito M, Itoh M and Tomoda A: Combined treatment with bortezomib plus bafilomycin A1 enhances the cytocidal effect and induces endoplasmic reticulum stress in U266 myeloma cells: crosstalk among proteasome, autophagy-lysosome and ER stress. Int J Oncol 387: 643-654, 2011.

28. Matsuyama S and Reed JC: Mitochondria-dependent apoptosis and cellular $\mathrm{pH}$ regulation. Cell Death Differ 7: 1155-1165, 2000.

29. Matsuyama S, Liopis j, Deverqux QL, Tsien RY and Reed JC: Changes in intramitochondrial and cytosolic $\mathrm{pH}$ : early events that modulate caspase activation during apoptosis. Nat Cell Biol 2: $318-325,2000$.

30. Shirato K, Imaizumi K, Abe A and Tomoda A: Phenoxazine derivatives induce caspase-independent cell death in human glioblastoma cell lines, A-172 and U-231 MG. Oncol Rep 17: 201-208, 2007.

31. Takasaki A, Hanyu H, Iwamoto T, Shirato K, Izumi R, Toyota H, Mizuguchi J, Miyazawa K and Tomoda A: Mitochondrial depolarization and apoptosis associated with sustained activation of c-jun-N-terminal kinase in the human multiple myeloma cell line U266 induced by 2-aminophenoxazine-3-one. Mol Med Rep 2: 199-203, 2009

32. Miyano-Kurosaki N, Ikegami K, Kurosaki K, Endo T, Aoyagi H, Hanami M, Yasumoto J and Tomoda A: Anticancer effects of phenoxazine derivatives revealed by inhibition of cell growth and viability, disregulation of cell cycle, and apoptosis induction in HTLV-1-positive leukemia cells. J Pharmacol Sci 110: 87-97, 2009.

33. Miyano-Kurosaki N, Kurosaki K, Hayashi M, Takaku H, Hayafune M, Shirato K, Kasuga T, Endo T and Tomoda A: 2-Aminophenoxazine-3-one suppresses the growth of mouse malignant melanoma B16 cells transplanted into C57BL/6Cr Slc mice. Biol Pharm Bull 29: 2197-2201, 2006.

34. Hongo T, Miyano-Kurosaki N, Kurosaki K, Hata A, Harigae S and Tomoda A: 2-Aminophenoxazine-3-one prevents pulmonary metastasis of mouse B16 melanoma cells in mice. J Pharmacol Sci 114: 63-68,2010.

35. Tamura J, Tanaka J, Fujita K, Yoshida M, Kasamatsu T, Arii S and Tobe T: Effect of anticancer agents on cell cycle of regenerating hepatocytes in rats. J Surg Res 53: 218-226, 1992. 